\title{
An investigation into the prevalence of Combination Syndrome
}

Short title: Prevalence of Combination Syndrome.

Dr Rita Bagga, ${ }^{a}$ BDS, MJDF, DPDS, MSc, PGCert, FHEA, Professor Nigel D. Robb, ${ }^{\mathrm{b}}$ BDS, $\mathrm{PhD},{ }^{\mathrm{c}}$ Professor Michael R Fenlon, MA, PhD, BDentSc, FDS, FHEA,

Dr Rita Bagga, ${ }^{\text {a }}$ BDS, MJDF, DPDS, MSc, PGCert, FHEA, Clinical Teacher, King's College London Faculty of Dentistry, Oral \& Caraniofacial Sciences, Floor 26, Guy’s Hospital, London SE1 9RT. Email: rita.bagga@kcl.ac.uk

Professor Nigel D. Robb, ${ }^{\mathrm{b}}$ BDS, PhD, Professor of Restorative Dentistry, School of Dentistry and Oral Health, Gold Coast Campus, Griffith University, Qld 4222, Australia. Email: n.robb@griffith.edu.au

\footnotetext{
${ }^{c}$ Professor Michael R Fenlon, MA, PhD, BDentSc, FDS, FHEA,

Professor of Prosthodontics, King's College London Faculty of Dentistry, Oral \& Caraniofacial Sciences, Floor 22, Guy’s Hospital, London SE1 9RT. Email: michael.fenlon@kcl.ac.uk
}

Corresponding author: Professor Michael R Fenlon, Professor of Prosthodontics, King's College London Faculty of Dentistry, Oral \& Caraniofacial Sciences, Floor 22, Guy's Hospital, London SE1 9RT. Email: michael.fenlon@kcl.ac.uk. Telephone: (44) 02082445835.

Keywords: Combination syndrome, removable partial denture, complete denture 


\section{INTRODUCTION}

The first research evidence supporting the existence of Combination Syndrome was provided in 1972 [1]. Resulting from a study following 6 patients with mandibular anterior teeth opposing maxillary complete dentures over 3 years, Kelly identified five changes that he proposed constituted Combination Syndrome. These were: loss of bone from the anterior part of the maxillary ridge, overgrowth of the tuberosities, inflammatory papillary hyperplasia in the hard palate, extrusion of the lower anterior teeth and loss of bone under the denture bases.

Saunders et al. [2] suggested six more features supposedly associated with Combination Syndrome. These were: loss of vertical dimension, occlusal plane discrepancy, anterior spatial repositioning of the mandible, poor adaptation of prostheses, epulis fissuratum and periodontal changes. However, except for periodontal changes, these features probably represent poor Prosthodontic care rather than evidence of a syndrome. For these reasons they have been considered as of secondary importance in this study.

Shen \& Gongloff [3] in a study of 150 partially dentate and edentulous patients identified 25 patients with maxillary conventional complete dentures opposing mandibular anterior teeth, with or without mandibular distal extension removable partial dentures. Of the 25 patients examined 6 patients (24\%) displayed all five features of Combination Syndrome as described by Kelly [1]

Palmqvist et al [4] in a comprehensive review of the literature in relation to Combination Syndrome concluded that Combination Syndrome did not meet the requirements for a syndrome as commonly understood in medicine and that there was not conclusive evidence that Combination Syndrome existed as a clinical entity. 
Subsequently, Salvador et al. [5] examined 44 patients with maxillary complete dentures opposing mandibular anterior teeth, 32 of whom had bilateral distal extension saddle mandibular removable partial dentures and 12 had unilateral distal extension saddle mandibular removable partial dentures. None of these displayed features of Combination Syndrome. However, of 32 patients wearing maxillary complete dentures opposing mandibular anterior teeth and bilateral distal extension removable partial dentures, $8(25 \%)$ showed three features of Combination Syndrome. $70 \%$ of patients demonstrated excessive resorption of the anterior maxillary sextant and $40 \%$ had tuberosity enlargement.

The studies supporting the existence of Combination Syndrome suggest a prevalence of $25 \%$ in the at-risk group. However, these studies are few, small and had significant limitations which were neither accounted for nor controlled.

Undoubtedly, treating patients edentulous in the maxilla with opposing mandibular anterior teeth is challenging. However, treatments to address or to avoid Combination Syndrome are proposed and routinely taught to Dentistry students and graduates all over the world. These often necessitate interdisciplinary approaches, involving specialist skills, taking much time and incurring considerable costs. Such treatments can include implants [6], precision attachments and selective impression techniques [7], linear occlusion techniques [8] or cusp sulci analysis [9].

Without conclusive evidence that Combination Syndrome exists, or, if it exists, it is not rare, such treatments raise an ethical question in relation to treating patients unnecessarily.

To investigate the prevalence of features of Combination Syndrome, the following research questions were formulated: 
- What is the prevalence of features of Combination Syndrome in a sample of patients referred to a School of Dentistry, who had complete dentures in the maxilla opposed by mandibular anterior teeth with or without mandibular partial dentures?

- Do mandibular partial dentures offer any benefit or disadvantage to patients with complete dentures in the maxilla opposed by mandibular anterior teeth?

For the purposes of testing these research questions the following null hypotheses were tested:

- That Combination Syndrome was not to be found.

- That mandibular distal extension partial dentures would not prevent the occurrence of Combination Syndrome. 


\section{METHOD}

Research ethics approval was granted for the cross sectional observational study through the UK Integrated Research Applications System.

All potential candidates for the study were patients referred to Guy's Hospital for treatment. Those who met the criteria for inclusion in the study were approached at their first attendance and the study was briefly described. Those who expressed interest in participating were given a detailed verbal description and an information sheet to take home. At the next visit (usually 2 weeks later) patients were again approached and those still willing to participate were recruited and signed their consent to participate.

Inclusion criteria:

- Patients attending Guys Hospital,

- wearing maxillary removable complete dentures opposed by mandibular anterior natural teeth, with or without mandibular removable partial dentures,

- aged 18 years to 100 years,

- English speaker and reader

- Capable of giving consent.

Exclusion criteria:

- Those not meeting the inclusion criteria

- Presence of maxillary teeth whether erupted or not

- Presence of mandibular molars or second premolars, whether erupted or not. 
- Patients not habitually wearing their maxillary removable complete dentures

- Patients unable to give consent

Sample size calculation was not possible because of the small numbers of previous studies. Based on a pragmatic calculation based on throughput and potential for recruitment, a target figure of 100 was chosen. Patients were recruited over a period of one year.

Data collection.

All patients were recruited and examined by one researcher (MF).

Gender, age and whether complete maxillary dentures were opposed by partial mandibular dentures was recorded. Time since loss of teeth was recorded.

Ridge form was scored for each of the five edentulous sextants using Cawood \& Howell's index [10]. The examiner previously demonstrated high levels of intra-operator reliability when using this index [11].

Excessive resorption of the anterior sextant of the maxilla was deemed to be present where the anterior sextant had a worse Cawood and Howell score than both posterior maxillary sextants.

Excessive resorption of the posterior sextants of the mandible was deemed to present where both posterior sextants had worse Cawood and Howell scores than both of their opposing posterior maxillary sextants. For the purposes of this research, where one sextant had a worse Cawood and Howell score than its opposing posterior maxillary sextant on the same side this was also recorded.

Presence or absence of extrusion of the mandibular anterior teeth was judged by the relationship of the cement-enamel junctions of the remaining teeth to the lower lip. If 
compensatory over eruption of these teeth had occurred, the gingival margin or the CEJ would be visible on smiling or at rest. This measure was chosen because the literature on overeruption identifies overeruption by relating the position of teeth to adjacent teeth in the same or opposing arches or to deviations from the curve of Spee. None of these approaches are applicable to isolated mandibular anterior teeth as the references normally used (other teeth) have been lost.

Anterior repositioning of the mandible where the position of maximum intercuspation was deemed to be more than $5 \mathrm{~mm}$ anterior to the first contact in retruded arc of closure.

Presence or absence of epulis fissuratum was noted.

Where resting vertical dimension with the maxillary complete denture in place gave an overclosed appearance, this was regarded as evidence of reduced resting vertical dimension.

Presence of denture related stomatitis was classified using Newton's classification [12]. Newton classified papillary hyperplasia as Grade III, the most severe form.

Periodontitis was deemed to be present if any probing depth greater than $3.5 \mathrm{~mm}$ was found using a WHO probe. Severity was classified as grade I $(3.5 \mathrm{~mm}-5.5 \mathrm{~mm})$, Grade II $(5.5 \mathrm{~mm}$ to $8.5 \mathrm{~mm})$ and grade III ( $8.5 \mathrm{~mm}$ or more $)$.

Where there was marked discrepancy of the plane of the maxillary residual alveolar ridge with the anterior being high (indicating resorption of the anterior maxillary ridge) and the posterior was low (indicating unresorbed or enlarged ridges in the tuberosity areas) this was noted.

Excessive maxillary alveolar ridge resorption in the anterior sextant was recorded. Presence of a displaceable maxillary anterior residual ridge was classified as grade I if there was any detectible displaceable tissue, grade II if the inferior half of the residual alveolar ridge was 
displaceable and grade III if all the bony ridge has been replaced with displaceable fibrous tissue.

Presence or absence of enlarged tuberosities and whether they were displaceable or firm was noted.

Patients were asked to grade how well their maxillary complete dentures fitted on a scale of 1 (worst), to 3 (best). Patients were asked to grade ability to chew with dentures on a scale of 1 (worst), to 3 (best). Patients were asked if their complete dentures caused pain on chewing. 
Statistical Methods.

Data were recorded on an Excel Spreadsheet (Microsoft Corporation, One Microsoft Way, Redmond 98052 Washington, USA). Data were analyzed using STATA (StataCorp LLC, 4905 Lakeway Drive, College Station, Texas 77845-4512, USA)

For comparison of distribution of age in women and men, the Kolmogorov-Smirnov test for equality of distribution was used.

Because all the remaining tests of data involved categorical data, Pearson's $\mathrm{X}^{2}$ test was used in all cases. Where numbers in individual cells in contingency tables were low (fell below 6), Fisher's Exact test was also used because it is more reliable than Pearson's $\mathrm{X}^{2}$ test in these circumstances. 


\section{RESULTS.}

A total of 100 patients consented to participate in the study and were examined. One patient withdrew from the study after the closing date for data collection so that patient's data were withdrawn, leaving data of 99 participants to be analysed.

54 women (mean age 70.4) and 45 men (mean age 71.3) participated. Distributions of ages for women and men were not found to be significantly different using the Kolmogorov-Smirnov test for equality of distribution $(\mathrm{K}-\mathrm{S}$ corrected $\mathrm{p}$ value $=0.106)$

Mean age of patients was 70.81 years, (SD 9.96; range $46-89$ )

Mean time since the maxillary teeth were lost was 16.14 years, (SD 12.36; range $3-63$ ).

Mean time since the last mandibular teeth were lost was 12.19 years, (SD 8.17; range $1-29$ ).

Mean number of sets of dentures (C. or C/P) was 2.7, (SD 1.6; range $1-10)$.

Mean age of dentures being worn was 5.84 years, (SD 5.37; range $1-30$ ).

Age of dentures was not significantly different $(\mathrm{P}=0.9785)$ between men $(5.82 \mathrm{yrs}$; SD 5.54) and women (5.85; SD 5.29).

Possible "Combination Syndrome" related features.

All 38 patients with excessive resorption of the anterior sextant of the maxilla had fibrous replacement of the bone to some extent. Of these, 19 were grade I, 13 were grade II and 6 were grade III.

Excessive resorption of the residual alveolar ridges in the posterior mandibular sextants was not found bilaterally in any case. However, unilateral excessive resorption of the mandibular alveolar ridge was deemed to be present in 13 patients where the Cawood \& Howell score for 
one mandibular sextant was worse than for the corresponding posterior maxillary sextant, indicating greater resorption than their opposing maxillary counterparts.

Extrusion of the mandibular anterior teeth was not found in any patient.

Papillary hyperplasia was found in two patients (out of a total of 7 with denture related stomatitis).

Two patients had enlarged tuberosities, in each case, bilateral enlargement of the fibrous displaceable type.

No patient was found to have more than two features of "Combination Syndrome" as described by Kelly

Eight patients were found to have two features of Combination Syndrome (2 patients with displaceable maxillary anterior ridges and enlarged tuberosities, 6 patients with increased resorption of the mandibular sextants and displaceable maxillary anterior ridges). There was a problem even with these numbers. In every case only one mandibular ridge scored worse than the maxillary ridge on the same side. In no case were both posterior mandibular residual ridges judged to have a worse Cawood and Howell score than their respective opposing maxillary ridge sextants. However, 17 patients were found to have less resorption of mandibular sextants when compared with the equivalent maxillary sextants.

38 patients displayed one feature of Combination Syndrome (30 patients with displaceable maxillary anterior ridges, and 7 patients with increased resorption of one or both mandibular sextants). 
No patients were found to have three or more Combination Syndrome features. Women were more likely to wear a complete maxillary denture opposing a mandibular partial denture than were men (Table 1).

Wearing a mandibular partial denture did not protect against having a displaceable maxillary anterior residual alveolar ridge, the trend suggested the opposite, but the difference was not statistically significant (Table 2).

No patients demonstrated anterior repositioning of the mandible, reduced resting vertical dimension or presence of epulis fissuratum so these factors were discounted. Two patients had deranged residual maxillary ridges planes but as both of these had enlarged tuberosities causing an antro posterior derangement of the occlusal plane, these also were discounted as double entries of the same condition (enlarged tuberosities).

A total of 55 patients were diagnosed as having periodontitis and an association between presence of periodontitis and displaceable maxillary anterior residual ridges was found (Table $3)$.

The presence of a mandibular partial denture was significantly associated with prevalence of periodontitis. (Pearson $\mathrm{X}^{2}=5.94 ; \mathrm{df}=3 ; \mathrm{P}=0.119$ ).

Tooth condition was not associated with wear or non-wear of mandibular partial dentures $\left(\right.$ Pearson $\mathrm{X}^{2}=0.3952 ; \mathrm{df}=2 ; \mathrm{P}=0.821$. Fisher's exact $\left.=0.86\right)$.

Fit of the maxillary complete denture was significantly positively associated with wearing mandibular partial dentures (Table 4).

Chewing ability as rated by patients was significantly positively associated with wearing mandibular partial dentures (Table 5). 
Pain from wearing the maxillary complete denture as reported by the patient was not associated with whether the complete maxillary denture was opposed by a mandibular partial denture or not $\left(\right.$ Pearson $\left.\mathrm{X}^{2}=2.6592 ; \mathrm{df}=1 ; \mathrm{P}=0.103\right)$.

Complete maxillary dentures not opposing mandibular partial dentures were significantly older than complete maxillary dentures opposing mandibular partial dentures $(\mathrm{C} /$ only mean $=7.63$ $\mathrm{yrs} ; \mathrm{SD}=1.26 ; \mathrm{C} / \mathrm{P}$ mean $=4.86 \mathrm{yrs}, \mathrm{SD}=0.44 ; \mathrm{P}=0.437)$. 


\section{DISCUSSION.}

Patients attending a Dental Hospital probably are not representative of the population as a whole. They are mostly referred by General Dental Practitioners because their problems are perceived as difficult for General Dental Practice or because efforts at treating these patients have been unsuccessful, as was the case with over 60 of the patients in the current study. All patients recruited were attending to have replacement dentures made, so some aspect of existing dentures surveyed had been judged to be unsatisfactory. This would explain the high proportion of unsatisfactory dentures. Thus, the sample was recruited from the potentially skewed Dental Hospital population. Recruitment within a Dental Hospital would be expected to identify a higher proportion of difficult cases, reflecting an exaggerated prevalence. Despite this bias, no cases of "Combination Syndrome" were found.

Considering Kelly's five signs of "Combination Syndrome" the findings were as follows:

- In no case were both posterior mandibular residual ridges judged to have a worse Cawood \& Howell score that their respective opposing maxillary ridge sextants

- Extrusion of the mandibular anterior teeth was not found in any patient.

- Papillary hyperplasia was found in two patients (out of a total of 7 with denture related stomatitis).

- Two patients had enlarged tuberosities, in each case bilateral enlargement of the fibrous displaceable type.

- 38 patients had displaceable fibrous replacement of the maxillary anterior ridges (22 grade I, 13 grade II and 3 grade III). 
No patient was found to have more than two features of "Combination Syndrome" as described by Kelly [1]. This finding undermines the notion that "Combination Syndrome" as described by Kelly exists.

Four patients were found to have two features of "Combination Syndrome" (two with displaceable maxillary anterior ridges and enlarged tuberosities and two with displaceable maxillary anterior ridges and papillary hyperplasia. This figure would rise to 10 if the six patients with increased resorption of the mandibular sextants on one side only and displaceable maxillary anterior ridges were included. However, it would not be appropriate to include these six patients. Increased bilateral resorption of the mandibular edentulous posterior sextants was not found in any case. Furthermore, 29 patients were found to have less resorption of mandibular posterior sextants when compared with their equivalent maxillary sextants. This suggests that factors other than those supposedly causative of Combination Syndrome are determinants of resorption in these sextants.

37 patients displayed one feature of Combination Syndrome (30 patients with displaceable maxillary anterior ridges, and 7 patients with increased resorption of one or both mandibular sextants - increased bone resorption of the anterior maxillary sextant, accompanied in all cases with displaceable fibrous replacement of maxillary anterior residual alveolar ridge.

Periodontitis is caused by the interaction between bacterial plaque and the host response, not by dentures. However, the significant association between the presence of periodontitis affecting the mandibular teeth and fibrous replacement of the anterior maxillary ridge might either be a chance finding or one could speculate that this is the expression of a tendency for bone to be lost in response to different insults to bone, inflammation associated with periodontitis process around teeth and possibly trauma from overloading of the maxillary 
denture. However, prevalence of periodontitis was significantly associated with wearing mandibular partial dentures. This finding was not surprising as partial dentures might be expected to make patients more susceptible to periodontitis given the plaque retentive nature of partial dentures. Patients with mandibular partial dentures rated their chewing ability significantly better than those without mandibular partial dentures. This quality of life benefit came at an increased risk of periodontitis.

Denture related stomatitis, of which papillary hyperplasia is the most severe type has been demonstrated to be caused by the denture biofilm interacting with the patient and not by trauma from occlusion [13]. If trauma from occlusion were implicated, mandibular complete denture bearing areas would be more prone to denture related stomatitis than maxillary complete denture bearing areas.

It is not obvious why the findings of this study are so much at variance with those of the three previous studies that found "Combination Syndrome" $[1,3,5]$. These studies involved small numbers, the combined number of patients in all three studies was at 75 , less than the 99 patients whose data were analyzed in this study.

\section{CONCLUSIONS.}

Combination Syndrome as classically described was not found in any of 99 patients drawn from a population skewed in favour of finding less common conditions. Evidence from this study suggests that Combination Syndrome either is rare or does not exist.

Mandibular partial dentures did not protect against development of excessive bony resorption in the maxillary anterior sextant. 
Wearing a mandibular partial denture opposing the maxillary complete denture seemed to improve satisfaction in these patients but increased periodontitis risk.

The presence of maxillary complete dentures opposed by mandibular anterior teeth with or without mandibular partial dentures was associated with a high prevalence of excessive bony resorption of, and fibrous replacement of the maxillary anterior residual alveolar ridge.

Declaration of Interest: None.

\section{Acknowledgements}

The authors acknowledge the Guys's \& St Thomas' Hospitals Trust for Research \& development approval and for permission to recruit subjects for this research project.

The authors acknowledge the research ethics review and approval for this research project involving human subjects completed by the Proportional Review subcommittee of South London Research Ethics Committee Number 3 (Research Ethics approval reference: 11/LO/0106). 


\section{REFERENCES}

1. E. Kelly, Changes caused by a mandibular removable partial denture opposing a maxillary complete denture. J. Prosthet. Dent. 27 (1972) 140 -150.

2. T.R. Saunders, R.E. Jr Gillis, R.P. Desjardins, The maxillary complete denture opposing the mandibular bilateral distal-extension partial denture: treatment considerations. J. Prosthet. Dent. 41 (1979) 124-128.

3. K. Shen, R.K. Gongloff, Prevalence of the 'Combination Syndrome' among denture patients. J. Prosthet. Dent. 62 (1989) $642-644$.

4. S. Palmqvist, G.E. Carlsson, B. Owall, Combination Syndrome: A literature review. J. Prosthet. Dent. 90 (2003) 270-275.

5. M.C. Salvador, A.L. do Valle, M.C. Ribeiro, J.R. Pereira, Assessment of the prevalence index on signs of Combination Syndrome in patients treated at Bauru School of Dentistry, University of Sao Paulo, J. Appl. Oral Sci. 15 (2007), 9-13.

6. P. Carlino, F. Pettini, C. Stefania, A. Ballini, F.R. Grassi, V. Pepe, Surgical and Prosthetic Rehabilitation of Combination Syndrome. Case Rep. Dent. 2014, Article ID 186213, 4 pages.

7. C.D. Lynch, P.F. Allen, The 'Combination Syndrome' revisited. Dent. Update. 31 (2004) $410-412$.

8. Jameson, WS.. The use of linear occlusion to treat a patient with Combination Syndrome: a clinical report. J. Prosthet. Dent. 85 (2001) 15-19.

9. S.M. Schmitt, Combination Syndrome: a treatment approach. J. Prosthet. Dent., 54 (1985) 664-671. 
10. J.I. Cawood, R.A. Howell, A classification of the edentulous jaws. Int. J. Oral \& Maxillofacial Surgery 17 (1988) 232-6.

11. M.R. Fenlon, M. Sherriff, J.D. Walter. Operator agreement in the use of a descriptive index of edentulous alveolar ridge form. Int. J. Oral Surg. 28 (1999) 38-40.

12. A.V. Newton, Denture sore mouth. Br. Dent. J. 112 (1962) 357-359.

13. M. Mantzourani, S.C. Gilbert, M. Fenlon, D. Beighton. Non-oral bifidobacteria and the aciduric microbiota of the denture plaque biofilm. Mol. Oral Microbiol. 25 (2010)190-9. 
Table 1. C/ only and C/P usage by gender.

\begin{tabular}{|l|l|l|l|}
\hline Gender & C/only & C/P & Total \\
\hline Male & 22 & 23 & 45 \\
\hline Female & 13 & 41 & 54 \\
\hline Total & 35 & 64 & 99 \\
\hline
\end{tabular}

Pearson $\mathrm{X}^{2}=6.6133 ; \mathrm{df}=1 ; \mathrm{P}=0.01$. 
Table 2. Displaceable ridge classification for maxillary complete denture only wearers and for maxillary complete denture and mandibular partial denture wearers.

\begin{tabular}{|l|l|l|l|l|l|}
\hline Denture & $\begin{array}{l}\text { Displaceable } \\
\text { wear }\end{array}$ & $\begin{array}{l}\text { Displaceable } \\
\text { ridge 1 }\end{array}$ & $\begin{array}{l}\text { Displaceable } \\
\text { ridge 2 }\end{array}$ & $\begin{array}{l}\text { Displaceable } \\
\text { ridge 3 }\end{array}$ & Total \\
\hline C/ only & 22 & 8 & 3 & 2 & 35 \\
\hline C/P & 39 & 11 & 10 & 4 & 64 \\
\hline Total & 61 & 19 & 13 & 6 & 99 \\
\hline
\end{tabular}

Pearson $\mathrm{X}^{2}(\mathrm{df}=3)=2.1677 \mathrm{P}=0.538$. Fisher's exact $=0.579$ 
Table 3. Presence of and severity of periodontitis was associated with presence and severity of displaceable maxillary anterior ridge.

\begin{tabular}{|l|l|l|l|l|l|}
\hline Periodontitis & Displaceable & Displaceable & Displaceable & Displaceable & Total \\
\hline 0 & ridge grade 0 & ridge grade 1 & ridge grade 2 & ridge grade 3 & \\
\hline 1 & 32 & 8 & 2 & 2 & 44 \\
\hline 2 & 16 & 8 & 1 & 1 & 26 \\
\hline 3 & 8 & 6 & 9 & 0 & 23 \\
\hline Total & 61 & 0 & 1 & 0 & 6 \\
\hline
\end{tabular}

Pearson $X^{2}=24.0376 ; \mathrm{df}=9 ; \mathrm{P}=0.004$. Fisher's exact $=0.004$ 
Table 4. Fit of $\mathrm{C} /$ with and without /P.

\begin{tabular}{|l|l|l|l|l|}
\hline Denture worn/ & 1 (worst) & 2 & 3 (best) & Total \\
\hline C/ & 14 & 12 & 9 & 35 \\
\hline C/P & 11 & 16 & 36 & 64 \\
\hline Total & 25 & 29 & 45 & 99 \\
\hline
\end{tabular}

Pearson $\mathrm{X}^{2}=6.6133 ; \mathrm{df}=1 ; \mathrm{P}=0.008$. Fishers exact $=0.007$. 
Table 5. Chewing ability comparing $\mathrm{C} /$ only and C/P.

\begin{tabular}{|l|l|l|l|l|}
\hline Denture worn/ & 1 (worst) & 2 & 3 (best) & Total \\
\hline Cl & 18 & 6 & 11 & 35 \\
\hline C/P & 13 & 12 & 39 & 64 \\
\hline Total & 31 & 18 & 50 & 99 \\
\hline
\end{tabular}

Pearson $\mathrm{X}^{2}=10.9293 ; \mathrm{df}=2 ; \mathrm{P}=0.004$. 